\title{
Adaptive Vibrarthographic Signal Denoising via Ant Colony Optimization Using Dynamic Denoising Filter Parameters
}

\author{
Rui Gong ${ }^{1,2, *}$, Kazunori Hase ${ }^{3}$, Hajime Ohtsu ${ }^{1,4}$, Susumu Ota ${ }^{5}$ \\ ${ }^{1}$ Graduate School of Systems Design, Tokyo Metropolitan University, Tokyo, Japan \\ ${ }^{2}$ Informatics and Computer Education Center, Mejiro University, Tokyo, Japan \\ ${ }^{3}$ Faculty of Systems Design, Tokyo Metropolitan University, Tokyo, Japan \\ ${ }^{4}$ Japan Society for the Promotion of Science, Tokyo, Japan \\ ${ }^{5}$ Faculty of Rehabilitation and Care, Seijoh University, Nagoya, Japan \\ Received 17 October 2021; received in revised form 30 November 2021; accepted 01 December 2021 \\ DOI: https://doi.org/10.46604/ijeti.2021.8718
}

\begin{abstract}
This study proposes an ant colony optimization (ACO) denoising method with dynamic filter parameters. The proposed method is developed based on ensemble empirical mode decomposition (EEMD), and aims to improve the quality of vibrarthographic (VAG) signals. It mixes the original VAG signals with different white noise amplitudes, and adopts a hybrid technology that combines EEMD with a Savitzky-Golay (SG) filter containing the dynamic parameters optimized by ACO. The results show that the proposed method provides a higher peak signal-to-noise ratio (PSNR) and a smaller root-mean-square difference than the regular methods. The SNR improvement for the VAG signals of normal knees can reach $13 \mathrm{~dB}$ while maintaining the original signal structure, and the SNR improvement for the VAG signals of abnormal knees can reach $20 \mathrm{~dB}$. The method proposed in this study can improve the quality of nonstationary VAG signals.
\end{abstract}

Keywords: ant colony optimization, denoising, ensemble empirical mode decomposition, vibrarthographic signal

\section{Introduction}

Knee disorders are often caused by the damage to cartilages and menisci, which leads to pain and disability in daily life activities [1]. In most cases of knee injury or disorder, the affected knees generate odd vibration or sound signals, which are referred to as vibrarthographic (VAG) signals. Although VAG signals are also present in healthy knees, the VAG signals from normal knees are regular and show insignificant amplitude. In contrast, the VAG signals from abnormal knees have features that are different from those of normal knees. Signal analysis technology can extract the features from abnormal VAG signals [2]. Notably, there is a long history of applying the vibration and sound of knee joints to the clinical diagnosis of knee disorders. Stethoscopes and palpation were used for diagnosis in the past, but the accuracy depends entirely on doctors' experience. With the development of electronic technology, VAG signals can be digitized for data analysis. After signal processing and analysis, the damaged knee joints and healthy knee joints usually can be distinguished by extracting the features of VAG signals. However, it is challenging to analyse VAG signals that contain non-periodic and fast transient features [3].

Some researchers argued that wavelet transform (WT) is useful for extracting the signals captured during dynamic knee movement. Indeed, WT has a higher accuracy resolution in terms of time and frequency scales than fast Fourier transform (FFT). The reason is that FFT decomposes a signal into its frequency components and loses information in the time domain. The continuous WT analysis is also considered to be a tool for the VAG signal analysis of knee joint pathology [4].

* Corresponding author. E-mail address: gong-rui@ed.tmu.ac.jp

Tel.: +81-42-677-1801; Fax: +81-42-677-1801 
Before signal analysis, there is a challenge in pre-processing biological signals when it comes to denoising. The biological signals are susceptible to the surrounding environment and the physical state of participants. In addition, VAG signals are unstable signals with low signal-to-noise ratio (SNR), similar to sonar signals [5]. Although VAG signals usually can be used to draw a distinction between aged and healthy knees, the denoising process for VAG signals still affects the overall classification accuracy [6]. Hence, a better pre-processing model for denoising VAG signals is required. Compared with the denoising process for electrocardiogram (ECG), the denoising process for VAG signals is harder because there are few fixed-shape waveforms and inconspicuous peaks and valleys in VAG signals [7]. The VAG signal waveforms vary significantly, depending on whether the knee joints are affected by cartilage or ligament disorders [8]. The most problematic VAG signals in the denoising process are from healthy and slightly damaged knees. The VAG signals in these knees have fewer peaks and lower amplitudes, and are more easily disturbed by environmental interference and random noise. Many studies have attempted to remove the noise by traditional filters, but it is still difficult to distinguish signals from noise in VAG signals [6].

Therefore, this study develops a novel hybrid method that uses signal decomposition followed by filtering with optimal adaptive parameters. The representative decomposition method is empirical mode decomposition (EMD) method, which is often used to decompose biological signals and to combine well-designed filters for denoising. However, there is a mode-mixing problem that cannot be ignored [9]. The mode-mixing problem in VAG signals is caused by noise interference. There is a high-frequency noise discontinuity distribution in the original VAG signals, leading to make the signals to be localized high-frequency signals. As a result, the mode-mixing problem causes the point of the local extreme value of the original signal distribution to mutate, and trigger the envelope formed by the local extreme value point to exhibit jump phenomena. The resulting time-scale fit error causes a mode-mixing phenomenon occurred in the EMD process. When the mode-mixing phenomenon occurs, an intrinsic mode function (IMF) can cease to have physical meaning by itself, falsely suggesting that different physical processes are represented in a mode. Hence, an ensemble EMD (EEMD) method is developed as a modified method of EMD [10].

However, even with the use of EEMD-based hybrid denoising technology, the SNR improvement is not perfect for the VAG signals of healthy knees when the filter is with fixed thresholds and parameters. The denoising problem of abnormal VAG signals needs to be addressed as a priority. Therefore, researchers have sought help from evolutionary algorithms [11]. Some studies reported that a hybrid filter with a genetic algorithm (GA) has been successfully applied to ECG signals [10]. Nevertheless, there has been little progress in the noise reduction processing of VAG signals because of the inability to preserve real signal components while removing noise components. In addition, the noise components and real signals of VAG signals are not evenly distributed to each sub-signal by EEMD. Therefore, if a filter with adaptive thresholds can be added to each sub-signal, then it is possible to remove noise while preserving real signals to the greatest extent. An algorithm named zero-crossing rate (ZCR) is considered for verification. ZCR is the number of times within an epoch in which the signal crosses the zero-amplitude axis, to evaluate each sub-signal and the use of an optimized Savitzky-Golay (SG) filter to remove noise components. Thus, ant colony optimization (ACO) algorithm is used to select the optimal parameters in this study.

ACO algorithm as a swarm intelligence algorithm has been applied to several combinatorial optimization problems [12]. Ants deposit pheromones to mark a favourable path that other members can follow [13]. In the proposed method, the high SNR is the "food," and the parameters and threshold of a denoising filter are the "favourable path". Accordingly, this study attempts to design a denoising filter that combines ACO, and uses the optimization parameters based on EEMD to obtain stable VAG signals with high quality after denoising. In this study, the proposed method not only improves the VAG signal quality, but can also be applied to other low-amplitude biological signals based on the results of this study. The proposed method may become an inspiration for noise reduction technology for low-amplitude signals. The study is organized as follows. Section 2 describes the methods, section 3 provides the results and discussion, and section 4 contains the conclusions. 


\section{Methods}

\subsection{VAG signal acquisition and pre-processing}

A sensor (7BB-20-6L0 piezoelectric sensor, Murata) is attached to the tibia of participants, which is $15 \mathrm{~cm}$ below the center of the patella using medical double-sided adhesive tape. An acceleration sensor (KXR94-2050 tri-axis accelerometer, Kionix) is attached to the thigh of each participant to measure the flexion-extension angle of the knee joint. The participants must keep their arms tight against the body and keep their feet shoulder-width apart while sitting down on a calf-length chair with armrests. The patients stand up and sit down as steadily as possible three times within 3 seconds [14]. Before the experiment, each participant has practiced the required movement with the same rhythm. During the experiment, a device is used to record VAG signals and angle signals at a sampling rate of $2000 \mathrm{~Hz}$.

In the data pre-processing step, to monitor the features of the interest from VAG signals, the flexion-extension movement must be repeated three times to determine the timestamp of angle signals. The raw VAG signals are interrupted according to the timestamp, and each VAG signal length is set between 5000 and 6000.

Correcting the baseline wander is an essential step. A baseline wander is a typical artifact that causes the distortion of the recorded VAG signals and hinders the correct diagnosis of diseases [15]. The baseline wander is a low-frequency artifact in VAG signals that commonly arises from the amplifier or participant movement and is quickly removed by a moving-average filter in the present study. An example of the acquired VAG signals after cutting and correcting the baseline wander is shown in Fig. 1.

In Fig. 1(a), although the VAG signals in the left and right knees do not differ significantly in stages (a)-(b), more substantial amplitude peaks are present in the left knee in stages (b)-(c). These peaks have a higher full width at half maximum (FWHM), which is probably the reason of the slight pain. The possibility of injury may be hidden by pain or discomfort in the knee joint, manifested as different frequency and amplitude peaks (particularly high FWHM peaks) in the VAG signal waveform [16]. For the denoising process, the signals without any particularly pronounced peaks are more difficult to handle. The present study prefers to use the normal knee VAG signals to test the denoising capability of the proposed algorithm.

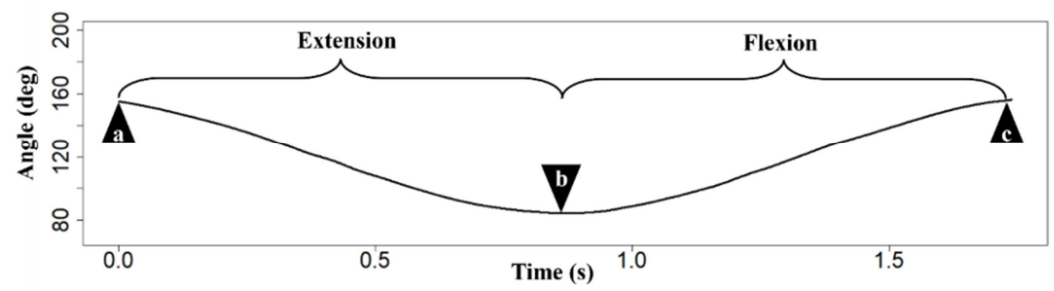

(a) Standing-up movement and sitting-down movement

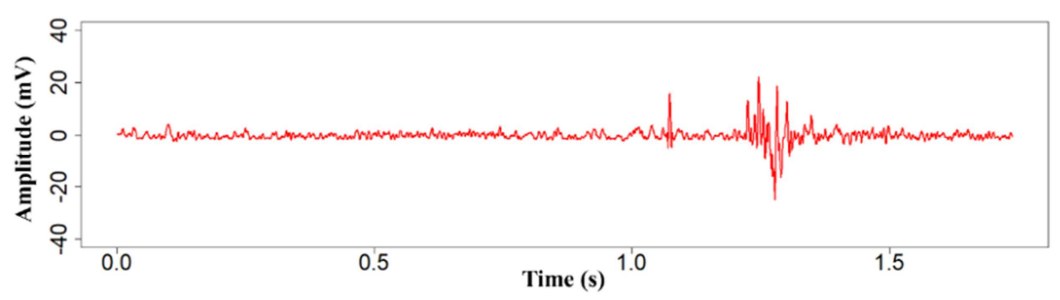

(b) VAG signals obtained from the left knee of a participant

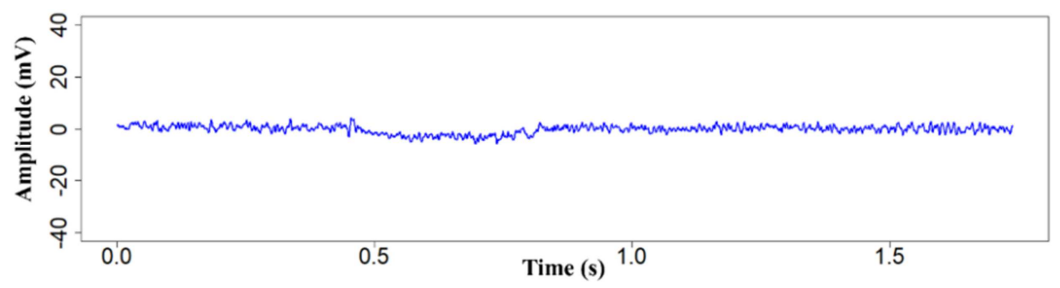

(c) VAG signals obtained from the right knee of a participant

Fig. 1 VAG signals and bending angles during dynamic knee movement 
Therefore, the present study uses 60 sets of VAG data obtained from 26 participants with normal knees. The participants are aged between 20 and 30, do not have any pain or discomfort in knees, and are not diagnosed with knee joint disorders before the experiment. For comparison, this study also uses 10 sets of VAG data obtained from three participants who are aged between 80 and 90 and have knee pain and limited mobility [6]. All the participants have provided informed consent, and the ethics review board of Tokyo Metropolitan University has approved the study design (approval number H2-111).

Before the denoising algorithm is used, white noise signals are added to the original VAG signals. White noise is a random signal with a constant power spectral density. The power of the white noise signals in each frequency band is the same, and is often used to test the effectiveness of the denoising method [17]. In the present study, white noise is added in two ways. The first is to set the white noise amplitude based on the SNR of the noisy VAG signals and the original VAG signals. The second is to set the white noise amplitude based on the percentage of the peak value of the original VAG signals. The white noise added in this study does not influence the results. It is only used to test the effectiveness of the denoising method in various imaginary-noise environments.

\subsection{Standard denoising method}

After completing the pre-processing work of all the collected VAG signals, the next stage is to conduct a denoising process. Denoising methods are usually classified into two types: a denoising process after decomposition and a direct denoising process, as shown in Fig. 2.

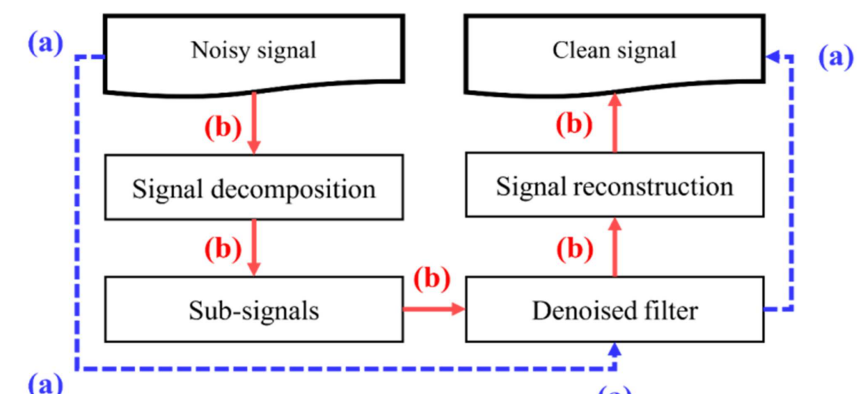

(a) A direct denoising process

(a)

(b) A denoising process after decomposition

Fig. 2 Denoising methods

As the name implies, a direct denoising process is performed on the original signals. In contrast, with the denoising process after decomposition, the original signals are firstly decomposed into sub-signals, and then the denoising process is conducted on these sub-signals. The denoising process after decomposition (path (b) in Fig. 2) is chosen as the standard denoising method in this study. Because any signal can be assumed to consist of different sub-signals of an oscillation, the sum of the information from the sub-signals is greater than that from the original signals [18]. The proposed method not only decomposes VAG signals using EEMD, but also selects the decomposed sub-signals. Unlike the standard method, the proposed method uses the optimized filter parameters for different sub-signals.

\subsection{Proposed denoising method}

As shown in Fig. 3, the proposed method for the denoising process can be divided into three phases: decomposition, optimization, and denoising. In the decomposition phase, the noisy VAG signals are decomposed into several sub-signals by EEMD, and the classification of the sub-signals is conducted by ZCR. An additional note is that ZCR carries frequency information in the time domain, but does not change the frequency-domain components. The optimization phase involves finding and outputting the optimal parameters for the denoising filter. Finally, the denoising phase involves adapting the filters with optimal parameters to reduce the noise of each sub-signal and reconstruct clean VAG signals. 


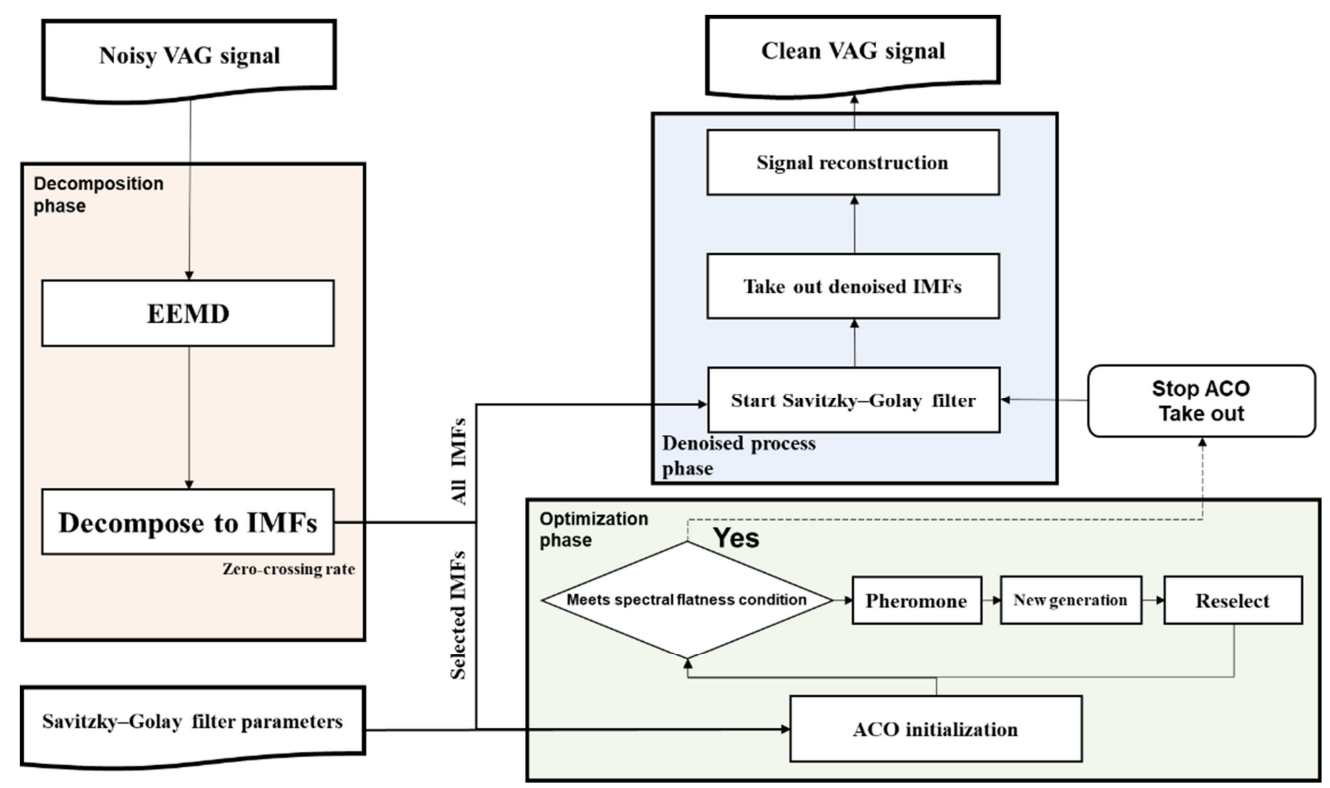

Fig. 3 Flowchart of the proposed ACO-EEMD hybrid technique for the denoising process

\subsubsection{Decomposition phase}

In this phase, the main task is to decompose the noisy VAG signals using the EEMD method. The EEMD algorithm can usually be described as:

$$
x^{i}[n]=x[n]+G^{i}[n]
$$

where the target signal $x[n]$ and $G^{i}[n](i=1, \ldots, I)$ are different realizations of white Gaussian noise. The standard deviation $\widehat{m}$ of white Gaussian noise $G^{i}[n]$ is relative to the absolute amplitude of $x[n]$. This study multiplies the absolute amplitude using a multiplier to denote the noise width. The multiplier in this study is $m=0.05$, which is lower than the recommended value of $m=0.2$ [19].

$$
\hat{m}=m \cdot|\max (x[n])-\min (x[n])|
$$

The crucial components of VAG signals are mainly concentrated at high frequencies. Consequently, the filtered high-frequency white Gaussian noise strength must be reduced to detect VAG signals. Therefore, the threshold is set for the maximum filtered white noise level. A 5\% absolute amplitude is chosen for noise standard deviation. In fact, the additional noise enhances the decomposition method to avoid mode-mixing and improve the performance of the EEMD algorithm. However, the impact outside the decomposition process is minimal after the ensemble average [19].

Each $x^{i}[n](i=1, \ldots, I)$ is completely decomposed by EMD, giving the modes $I M F_{k}^{i}[n]$ of the signals (where $k=$ $1, \ldots, K$ denotes the modes). This study defines $\overline{I M F_{k}}$ as the $k^{\text {th }}$ mode of $x[n]$, which is obtained as the average of the corresponding $I M F_{k}^{i}$ as follows:

$$
\overline{I M F_{k}}[n]=\frac{1}{I} \sum_{i=1}^{I} I M F_{k}^{i}[n]
$$

In the proposed method, the signals are decomposed into 10 IMFs using EEMD, and the deviation values of their ZCRs are calculated. The ZCR of a signal frame is the rate of signal changes of the signal during a frame, which reflects the number of times that the signal changes from positive to negative, divided by the length of the frame [20]. Letting $y_{i}(n)=0,1, \ldots, N-$ 1 be the sample of the $i^{\text {th }}$ frame, the ZCR is defined by the following equation: 


$$
\operatorname{ZCR}(i)=\frac{1}{2 N} \sum_{n=0}^{N-1}\left|\operatorname{sgn}\left[y_{i}(n)\right]-\operatorname{sgn}\left[y_{i}(n-1)\right]\right|
$$

where

$$
\operatorname{sgn}\left[y_{i}(n)\right]=\left\{\begin{array}{c}
1, y_{i}(n) \geq 0 \\
-1, y_{i}(n)<0
\end{array}\right.
$$

Then, all the deviation values of $Z C R(i)$ are arranged from large to small, and the five largest IMFs are labeled through $m f_{1}$ and $m f_{5}$. The five smallest IMFs are combined into one IMF, and the result is labeled as $i m f_{\text {combine }}$. In addition, a denoising filter must be prepared for the IMFs, where the SG filter is chosen as the base filter.

The SG filter is often used to smooth biological signals for the SNR improvement [21]. The SG filter is also referred to as the least-squares smoothing filter, which performs better for VAG signals than traditional moving-average filters, with a particular advantage in the high-frequency domain. The SG filter is not very good at rejecting noise, but is more effective for preserving high-frequency information components [22]. In addition, because the SG filter is particularly good at preserving the line shape, this filter is conducive to the signal reconstruction processing in the next step. Finally, the SG filter has few parameters that can reduce the optimal calculation time. Therefore, the SG filter is chosen for the present study.

\subsubsection{Ant colony optimization (ACO)}

Further explanation of ACO is needed to understand the process shown in Fig. 4. Ants will set out in groups to carry the food through the path without a bar. When the bar appears, the initial generation of ants will randomly choose a new path with a certain probability, but the pheromones remaining on the path will continue to decrease over time. The shorter path will have more pheromones remaining, and the selection probability will be more transparent towards this path, eventually reaching an optimal solution. Therefore, parameters such as the number of generations, number of ants per generation, pheromones, weights, and selection probabilities will appear in the next algorithm.

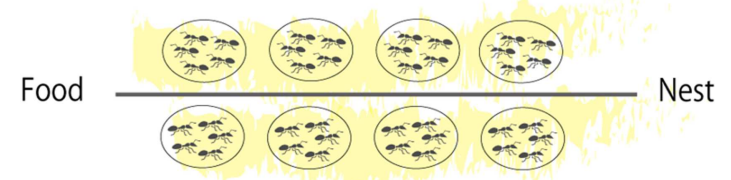

(a) Ants find no obstacles on the path

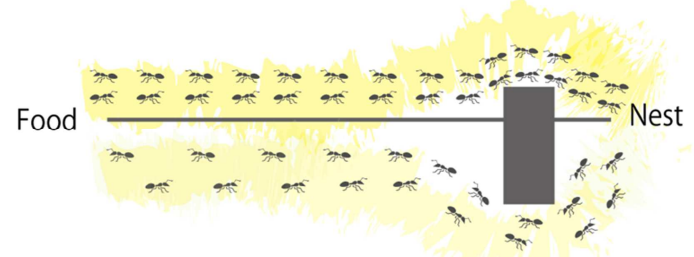

(c) More residual pheromones when choosing the shorter path

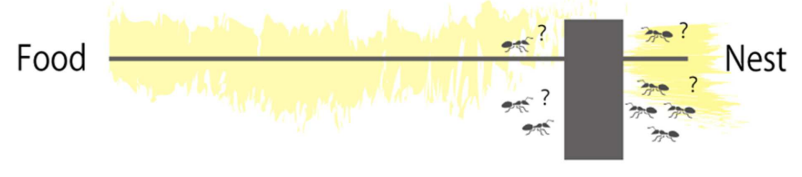

(b) Ants find obstacles on the path

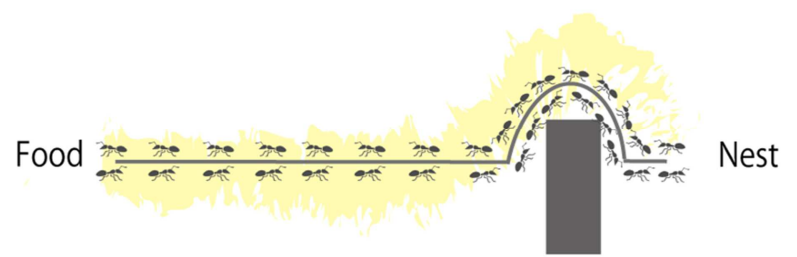

(d) Ants choose the shorter path

Fig. 4 Schematic diagram of ACO

\subsubsection{Optimization phase}

The SG filter is referred to as the objective function $\left(F_{\text {objective }}\right)$ of the ant colony system. There are two main dynamic parameters of $F_{\text {objective }}$ : the frame length $\lambda$ (which must be odd) and the polynomial order $\xi$. The dynamic parameters must be set within a reasonable range. In addition, there is a parameter that returns the $v_{t h}$ derivative of the convolution coefficients. The derivative order is set to zero for the filter to smooth the curve. 
The ACO algorithm continuously optimizes the dynamic parameters to achieve a better noise reduction effect. A condition function (called the fitness function in the evolutionary algorithm) must be manually set to accomplish this goal. The choice of the condition function is essential because this function directly affects the logical structure of the whole algorithm. In the present study, spectral flatness is chosen as a condition function $F_{\text {condition }}$ to plan the best path for the ant colony system. Typically, the spectral flatness is calculated by dividing the geometric mean into the power spectrum using the arithmetic mean. [23]. The spectral flatness is defined as follows:

$$
F_{\text {condition }}=\frac{\sqrt[N]{\prod_{n=0}^{N-1} H(n)}}{\frac{\sum_{n=0}^{N-1} H(n)}{N}}
$$

where $N$ represents the length of the power spectrum $H(n)$, and $n$ represents the value of a discrete sample of $H(n)$, where $n=1,2,3, \ldots, N$.

The combination of the condition and objective functions can export the cost function $F_{\text {cost }}$ to the ACO algorithm. The minimum value of the cost function $\min \left(F_{\text {cost }}\right)$ is also referred to as the favorable path of the ant colony system. Here, $F_{\text {cost }}$ is described as:

$$
F_{\text {cost }}=\sum_{i=1}^{5} F_{\text {condition }}\left(F_{\text {objective }}\langle\operatorname{imf}, \lambda, \xi\rangle\right)+F_{\text {condition }}\left(F_{\text {objective }}\left\langle i m f_{\text {combine }}, \lambda, \xi\right\rangle\right)
$$

where $\lambda \in[5,21]$ and $\xi \in[2,5]$. The term $\lambda$ is specified as a positive odd integer, and $\xi$ must be a positive integer that is smaller than $\lambda-1$; it can also be summarized as a two-dimensional search space with constraints in the ACO algorithm [13].

In the initialization of the ACO algorithm, some parameters must be adjusted to balance the computation speed and accuracy. This study prefers to control the number of generations of ants rather than the number of ants. As such, the $\delta=100$ ants are set in each generation, which are equivalent to 25 generations. After the pheromone path is randomly generated for the first generation of ants in each search space, the step solutions $s_{1}$ and $s_{2}$ for each ant must be computed based on $F_{\text {condition }}$. In the pheromone path for the first generation of ants, the solutions increase in order in the archive according to the quality (minimum pheromone evaporation) and are indicated by the rank as $l$. The probability prob $b_{l}$ of choosing the path for each ant as a prototype for the next generation is calculated by the weight $w_{l}$, and is consistent with a Gaussian distribution function, as follows:

$$
\begin{aligned}
& w_{l}=\frac{1}{q \delta \sqrt{2 \pi}} \exp \left[-\frac{(l-1)^{2}}{2(q \delta)^{2}}\right] \\
& \operatorname{prob}_{l}=\frac{W_{l}}{\sum_{1}^{\delta} w_{l}}
\end{aligned}
$$

where $q \in(0,1)$. When $q$ is small, the best-ranked solutions are strongly preferred, and when $q$ is large, the probability becomes more uniform. $q=0.2$ is set to adjust the probability towards a more uniform distribution.

The optimal parameters are obtained by the iteration of the better solutions, choosing $s_{1}$ as the prototype. This process is achieved by adding the standard deviations $\sigma_{l}^{i}$ of each solution. This process is referred to as the pheromone update in ACO [24]. However, the pheromones evaporate over time. If the pheromones are not updated by the next generation of ants, the 
pheromone path will decrease at a constant evaporation rate $\eta$. For $\eta>0$, which is the same for all dimensions (the higher the value of $\eta$, the lower the convergence speed of the algorithm), $\eta=0.5$. To establish the value of the standard deviation $\sigma_{l}^{i}$ at the construction step $i \in\{1,2\}$, the average distance is calculated from the chosen solution $s_{1}$ to other solutions in the archive, and this distance is multiplied by the evaporation rate $\eta$. The standard deviation is calculated as follows:

$$
\sigma_{l}^{i}=\eta \sum_{j=1}^{\delta} \frac{\left|s_{v}^{i}-s_{l}^{i}\right|}{j-1}
$$

After removing the same number of worst solutions, the total size of the solutions does not change. This process ensures that only the best solutions are maintained so that these solutions effectively guide the ants in the search process [25]. When all the generations of ants are exhausted or three consecutive generations of ants return to the same solutions, the loop will be stopped. Because $F_{\text {cost }}$ is the sum of all values of $F_{\text {condition, }}$, the optimal solutions of $F_{\text {cost }}$ can also be found as dynamic parameters. When the ACO process stops, the dynamic parameters need to be removed and returned to the SG filter to denoise each corresponding IMF. Finally, clean VAG signals are obtained by reconstructing each denoised IMF.

\subsubsection{Evaluation for the effectiveness of the proposed method}

SNR and signal structural similarity are typically used to evaluate the denoising process. In this study, the peak SNR (PSNR) method is also used to evaluate the denoising process. PSNR can be seen as the ratio between the maximum possible power of a signal and the power of the corrupting noise. Although PSNR is often used for two-dimensional image processing, it is also convenient for one-dimensional VAG signals.

Because VAG signals have few fixed-shape waveforms, low-amplitude signals are easily masked by high-amplitude noise. The mean squared error (MSE) measures the signal process quality of an estimator, which is also the noise power in PSNR. MSE can be defined as follows.

$$
M S E=\frac{1}{N} \sum_{i=1}^{N}\left(a_{i}-b_{i}\right)^{2}
$$

where $N$ is the number of signal data points, $a_{i}$ is returned by the process, and $b_{i}$ is the actual value for the data point $i$. MSE is always non-negative and yields a better result for values closer to zero.

The one-dimensional PSNR process can be defined by the peak value and MSE, as follows:

$$
P S N R=20 \cdot \log _{10}\left[\frac{M A X\left(a_{i}\right)}{\sqrt{M S E}}\right]
$$

The method of comparing signal structural similarity is mainly performed using the Pearson correlation coefficient (PCC). PCC is used to measure the strength of a linear association between two signals, where $r=1$ indicates a perfect positive correlation and $r=-1$ indicates a perfect negative correlation. The term $r$ is obtained as follows:

$$
r=\frac{\sum_{i}\left(c_{i}-\bar{c}\right)\left(d_{i}-\bar{d}\right)}{\sqrt{\sum_{i}\left(c_{i}-\bar{c}\right)^{2}} \sqrt{\sum_{i}\left(d_{i}-\bar{d}\right)^{2}}}
$$

where $c_{i}$ and $d_{i}$ are the individual original and processed signal data points indexed with $i$, and $\bar{c}$ and $\bar{d}$ are the corresponding signal mean values. 
The percentage root mean square difference (PRD) can distort the measurement between the reconstructed and original VAG signals. As a percentage for evaluating the distortion measurement performance (lower is better), PRD is defined as follows [26]:

$$
P R D=\sqrt{\frac{\sum_{i=1}^{N}\left(e_{i}-g_{i}\right)^{2}}{\sum_{i=1}^{N}\left(e_{i}\right)^{2}}} \times 100 \%
$$

where $e_{i}$ and $\mathrm{g}_{i}$ are the $i^{\text {th }}$ samples of the original and processed VAG signals, respectively.

\section{Results and Discussion}

The VAG signal waveforms after denoising using the standard and proposed methods are shown in Fig. 5. In Fig. 5, the waveform obtained using the standard method (Fig. 5(d)) is smoother than that obtained using the proposed method (Fig. 5(c)). However, the SNR of the waveform obtained using the proposed method is greater than that obtained using the standard method. This is because the waveform obtained using the standard method loses more details in the denoising process than the waveform obtained using the proposed method. As shown by the close-up (Fig. 5(e)), the waveform obtained using the proposed method has far more mini peaks than the waveform obtained using the standard method.

The results indicate that, for the denoising process, the proposed method retains more details (mini peaks) because the ACO algorithm does an excellent job of differentiating the real signal and the noise, unlike the standard method without any calculation or optimization.

In fact, adding white noise to only one type of amplitude is insufficient. It is also necessary to verify if the proposed method is capable of rejecting noise of various strengths. By adding white noise of various strengths, noisy VAG signals are obtained from 0 to $25 \mathrm{~dB}$. The signals are processed using the proposed method and the standard method, and the results of the mean value and standard deviation are shown in Fig. 6.

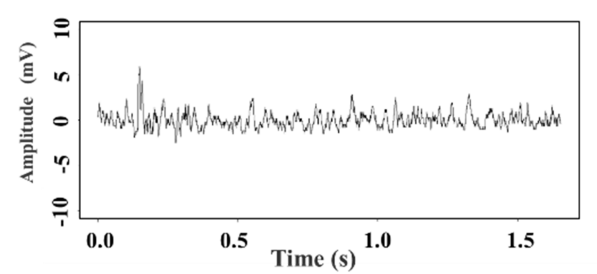

(a) Original signals

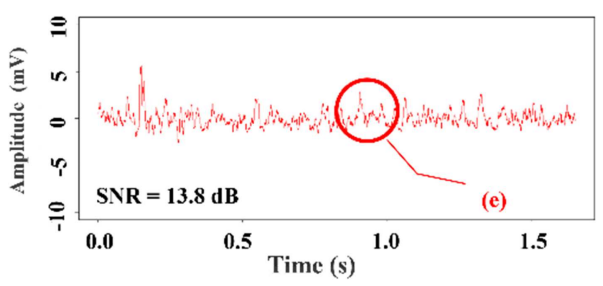

(c) Proposed method

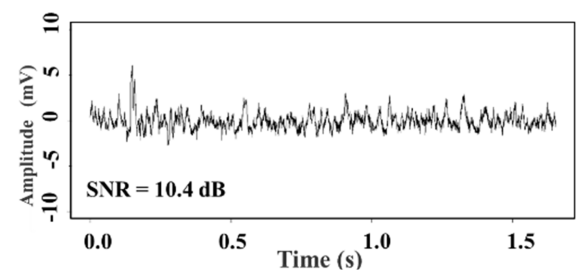

(b) Noisy signals

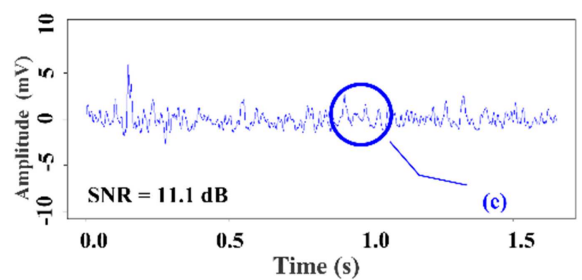

(d) Standard method

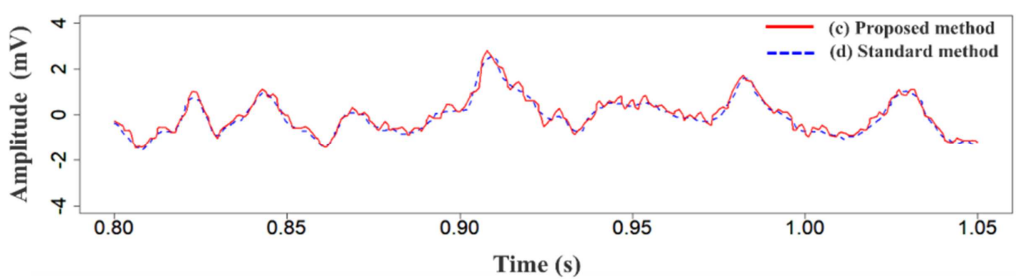

(e) Comparison between (c) and (d) after zooming

Fig. 5 Waveforms of the proposed method and the standard method 


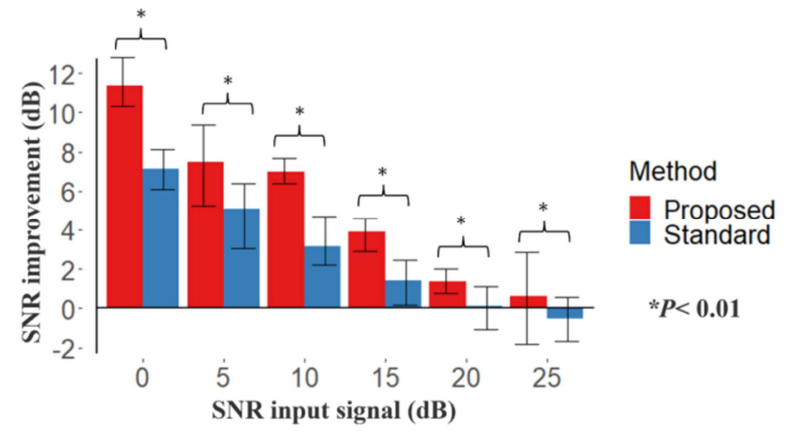

Fig. 6 SNR improvement of the proposed method and standard method

The proposed method is superior to the standard method in terms of SNR, as shown in Fig. 6. However, as the input signals' SNR increases, the improvement decreases significantly, either using the proposed or standard methods (for all $P$-values less than 0.01 and a maximum $P$-value of 0.0057 , by Student's t-test). When the SNR of the noisy input signals exceed $20 \mathrm{~dB}$, the improvement in the proposed method is almost negligible. However, the improvement in the standard method is negligible when the SNR of the noisy input signals exceed $15 \mathrm{~dB}$. Furthermore, the mean value of the improvement in the standard method is negative when the SNR of the noisy input signals is $25 \mathrm{~dB}$.

The main reason for the behavior of SNR is that the standard method is not capable of distinguishing between white noise and mini peaks. Conversely, in the proposed method, even though the mini-peaks and the white noise amplitude are very similar in the noisy VAG signals, the ZCR combined with EEMD can enhance the difference between mini-peaks and white noise by choosing the sub-signals. Moreover, the ACO algorithm optimizes the intensity of the denoising filter for each sub-signal based on the spectral flatness. Overall, the risk of the negative SNR improvement is effectively controlled using the proposed method. This advantage is also observed in PSNR, as shown in Fig. 7.

In general, a higher PSNR indicates that the signal reconstruction is of higher quality after denoising. Eq. (11) shows that PSNR is determined by the peak value and MSE as the information quality. The PSNR improvement can also be defined as a correction of information quality. As shown in Fig. 7, the PSNR improvement follows the same trend as the SNR improvement. The performance of the proposed method is always better than that of the standard method, and the performance improves as the white noise amplitude increases (for all $P$-values less than 0.01 and a maximum $P$-value of 0.0079 , by Student's t-test).

If VAG signals are intended for the clinical diagnosis of knee joints, the signal structure should not be changed significantly by signal processing [27]. Although the proposed method performs well in terms of noise reduction, its signal structure stability must be verified using PCC. As shown in Fig. 8, PCC is calculated between the original VAG signals and the clean signals obtained by the proposed method, between the original VAG signals and the clean signals obtained by the standard method, and between the original and noisy VAG signals.

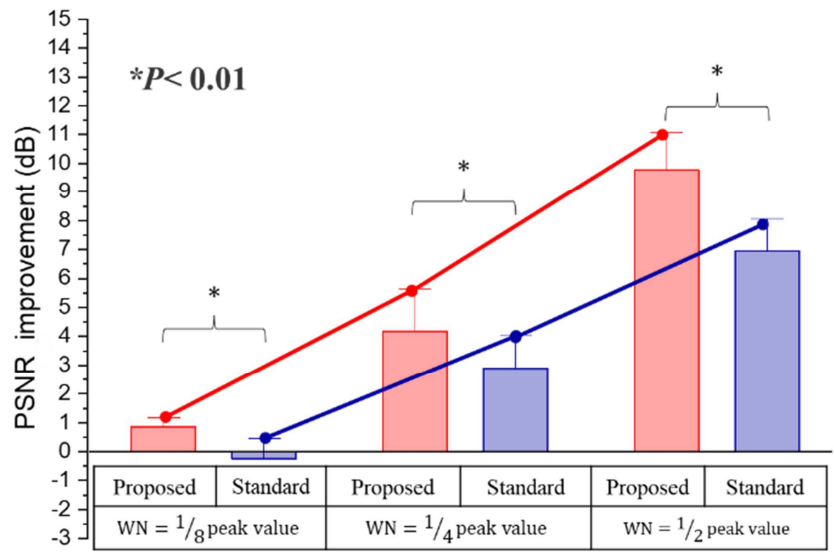

Fig. 7 PSNR improvement of the proposed method and standard method

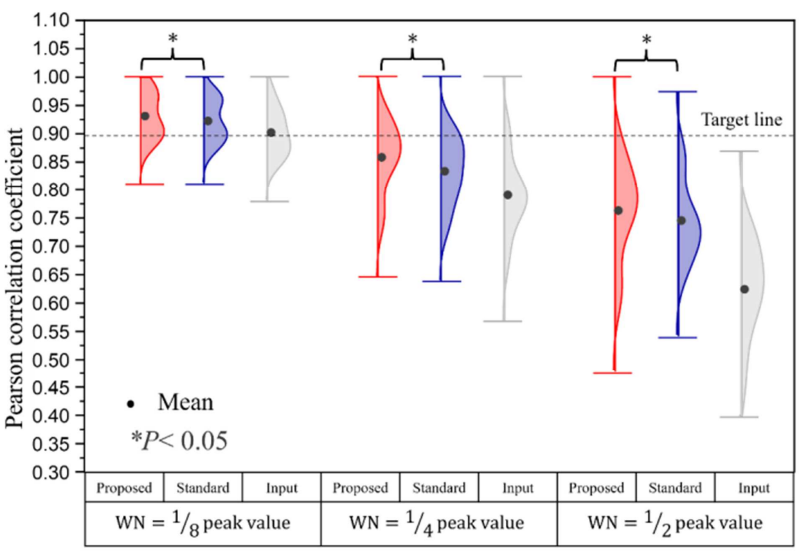

Fig. 8 Structural similarity between signals 
When low-intensity white noise is added, no significant increase in PCC is observed before or after the denoising process for either the proposed method or the standard method (for all $P$-values less than 0.05 and a maximum $P$-value of 0.044 , by Student's t-test). This is because the addition of white noise does not sufficiently affect the original signal structure. Following an increase in the white noise intensity, PPC increases after the denoising process, but does not overtake the target line. The signals before and after the denoising process, for which the waveforms are not the same, must have the same characteristics. Therefore, the structural similarity expectation (target line) is set to $90 \%$.

The VAG signal decomposition and signal reconstruction are suspected to affect the structural stability of VAG signals. Because VAG signals have several mini-peaks, the reconstructed mini-peaks can easily shift in the time domain. Therefore, this study uses various reference methods to verify this hypothesis.

An one-dimensional nonlocal means (NLM) filter and a discrete WT (DWT) filter are often applied to the denoising process of time-series biological signals, and can be quickly performed using MATLAB Toolbox [28-29]. NLM is a direct denoising process (path (a) in Fig. 2), and DWT is a denoising process after decomposition (path (b) in Fig. 2). Therefore, the filters with thresholds above are used for the contrast.

The noisy signals are mixed with 0,10 , and $20 \mathrm{~dB}$ white noise as the input signals for each denoising process. The present study uses four indicators to compare the improvement in the denoising process: SNR, PSNR, PCC, and PRD. The results of the four methods are presented in Fig. 9.

Overall, the results indicate that the proposed method and DWT denoising filter are the best performers among the four methods. Although the results for the proposed method are slightly worse than those for the DWT denoising filter for an SNR of $20 \mathrm{~dB}$ and a PCC of $0 \mathrm{~dB}$, the results for the proposed method are better than those for the other methods. This also indicates that the proposed method has room for improvement, especially with respect to the choice of filters with dynamic parameters. Since the denoising ability of the SG filter is improved by the hybrid technology, the proposed hypothesis is successfully verified. This conclusion has been reached because even if a signal with very high quality is input, the improvement can still be visible.

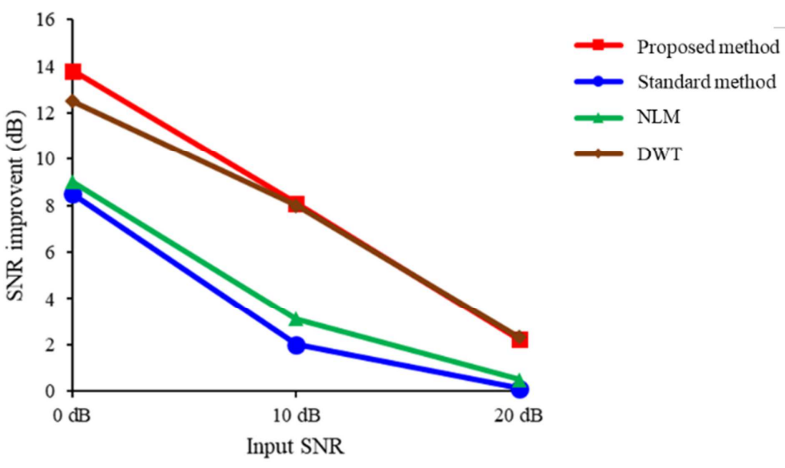

(a) SNR improvement of four methods

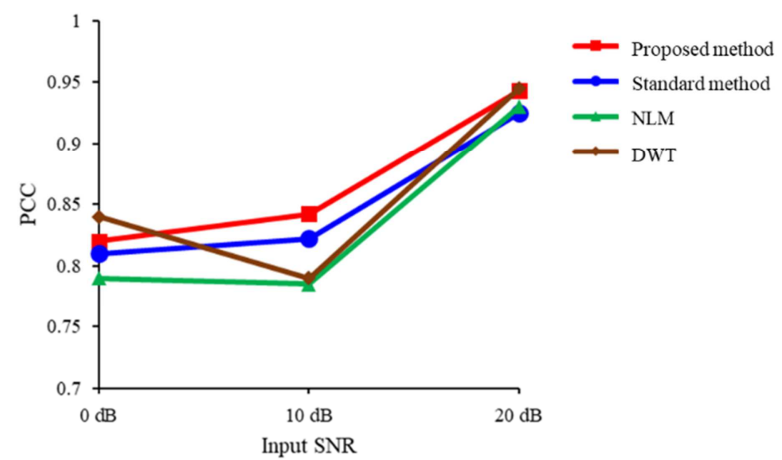

(c) PCC of four methods

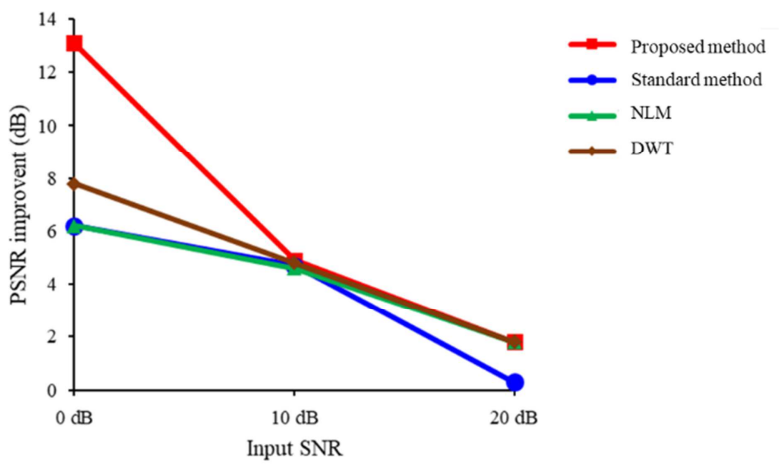

(b) PSNR improvement of four methods

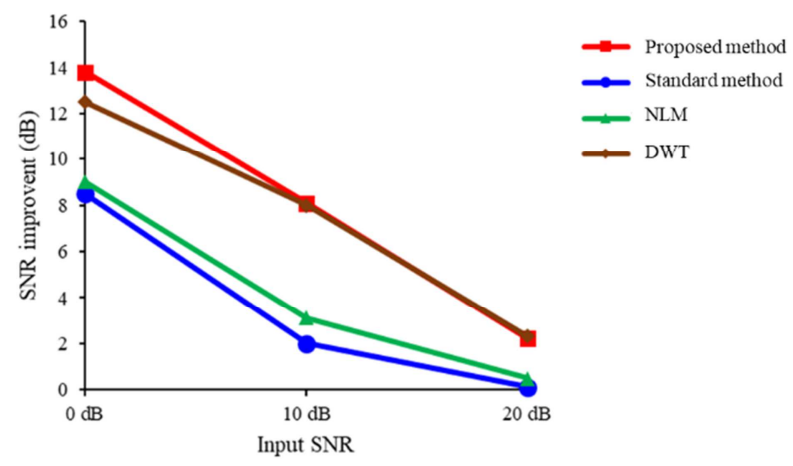

(d) PRD of four methods

Fig. 9 Denoising effects by the four methods examined in the present study 
In addition, the proposed method is different from the DWT method, which decomposes VAG signals using a high-pass filter and a low-pass filter [30]. The proposed method decomposes the signals using EEMD, which involves only amplitude components without frequency components. Because the ultimate goal of the present study is to apply VAG signals to the clinical diagnosis of knee disorders, the proposed method can guarantee the VAG signal quality while being beneficial for frequency-domain feature extraction in the next stage. Thus, the VAG signals of abnormal knees are collected, and the practicality of the proposed method is verified.

These VAG signals from abnormal knees are also converted into input signals by adding white noise, as mentioned previously. The VAG signals are subsequently processed for the denoising process using the standard and proposed methods. The SNR improvement is shown in Fig. 10(a).

In the present study, it is observed that the SNR improvement of the proposed method is more significant than those of the standard method for all SNR input signals of abnormal knees (for all $P$-values less than 0.05 and a maximum $P$-value of 0.027 , by Friedman test). Fig. 10(b) also indicates that the VAG signals of abnormal knees can more easily obtain an SNR improvement than the VAG signals of normal knees for both the proposed method and the standard method. The reason is that the signals have steeper slopes, and sharper peaks are more easily denoised. In addition, this study also compares the difference in SNR improvement between the VAG signals from abnormal and normal knees using the proposed method and the standard method. The performance of the proposed method is worse than that of the standard method only when the SNR of the input signals is $0 \mathrm{~dB}$. When the SNR of the input signals is $5,10,15,20$, and $25 \mathrm{~dB}$, the performance of the proposed method is better than or equal to that of the standard method. The analysis results are found to be significant at $5 \mathrm{~dB}, 15 \mathrm{~dB}$, and $25 \mathrm{~dB}$ of input signals after Friedman's test, and not significant at the rest. Fig. 11 shows a comparison of the VAG signals of abnormal knees before and after denoising.

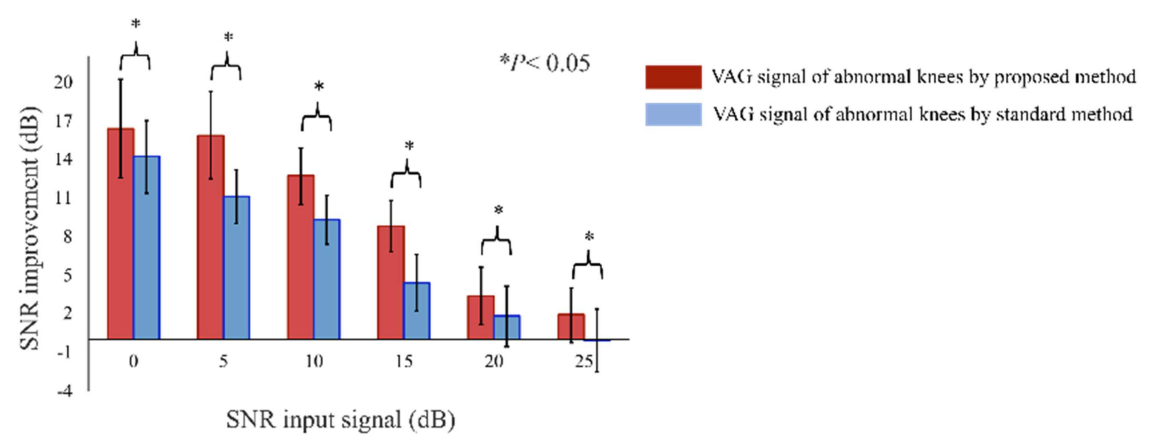

(a) SNR improvement of the VAG signals from abnormal knees by the proposed method and standard method

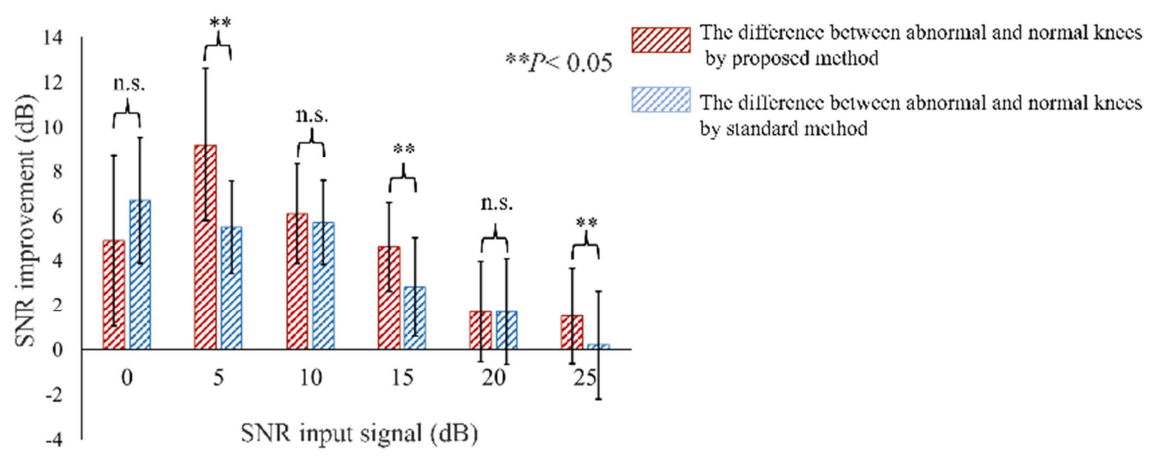

(b) Comparison between the SNR improvement of abnormal and normal knees by the proposed method and standard method

Fig. 10 SNR improvement of the VAG signals in abnormal and normal knees by the proposed method and standard method 


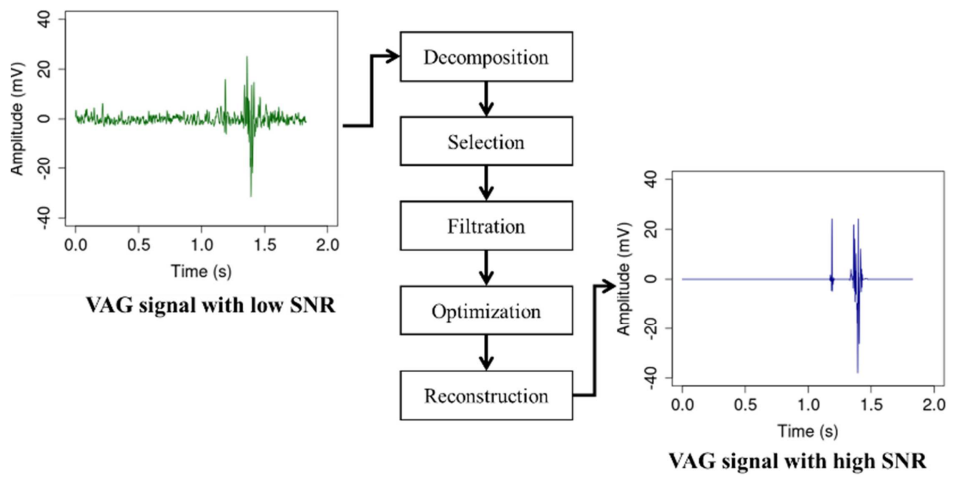

Fig. 11 VAG signals of abnormal knees before and after denoising

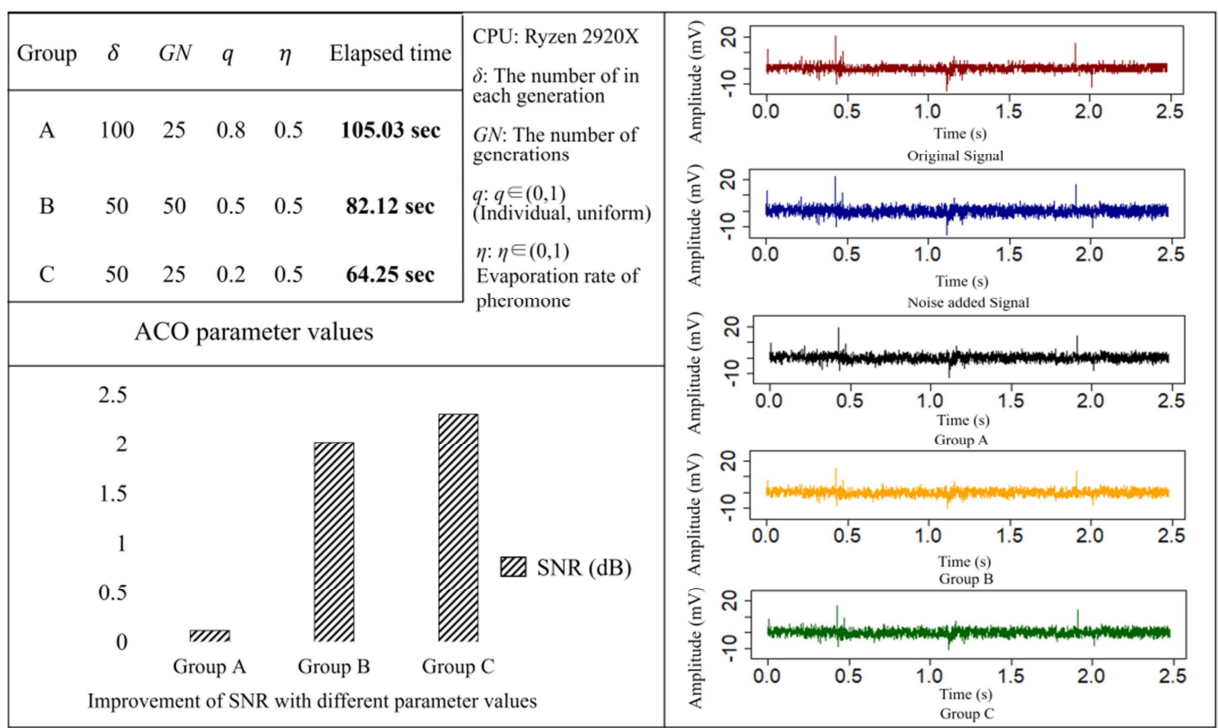

Fig. 12 Denoising effects by different parameter values of ACO

As can be seen in Fig. 11, the VAG signals of abnormal knees exhibits more aggressive denoising outcome than that of normal knees when the default ACO makes the filter optimization. Therefore, the parameters of ACO need to be adjusted to make it less reserved when processing the VAG signals of normal knees. Finally, a simple comparison for the noise reduction effects of different parameter values is made. The noisy VAG signals with a length of 5000 and an SNR of $5 \mathrm{~dB}$ are used as the input. The denoised results are presented in Fig. 12.

In the present study, it is observed that increasing each generation takes more time than increasing the number of generations in the proposed method, and too many ants or generations will not bring better effects. Conversely, the parameter that most affects the SNR improvement is $q$, which is of increased relevance to individuals or a uniform distribution. For VAG signals, the increased relevance to the uniform distribution can further improve the signal quality. It is expected that this recommended parameter value can be helpful when using the proposed method to process similar signals.

\section{Conclusions}

Noise interference in biomedical signals has become an obstacle to the advancement of medical diagnosis. Although some noise can be traceable, the source of most noise is uncertain. These uncertain noises may originate from environmental interference, non-target biological signals, or electronic components. To solve the noise problem, this study proposes an ACO denoising method. The conclusions of this study can be summarized as follows.

(1) In the present study, based on the hybrid technology of EEMD and the SG filter, a combined ACO algorithm denoising method is proposed, and the SNR improvement of VAG signals is verified. The results show that the proposed method can reject noise while ensuring that the real signals are retained. Overall, the denoising performance of the proposed method is 
better than that of the reference methods in terms of the VAG signals of either normal or abnormal knees. Furthermore, the proposed method significantly improves the stability of the VAG signal quality, and lays a foundation for the feature extraction and classification models.

(2) The hybrid technique proposed in this study has not been isolated but has grown with the advancement of computer technology in recent years. The outstanding performance of the proposed method relies on the ACO algorithm and powerful calculations for support. As computer technology continues to advance, the process of this hybrid filtering method will become more efficient. This will ensure that the denoising ability will become a key part to the advancement of biological signal processing technology.

(3) The proposed hybrid technology shows many benefits in this study, but there are still some limitations. Although EEMD solves the mode-mixing problem of EMD, there is room for improvement in the decomposition phase. Future studies should investigate how the decomposition and reorganization of signals can affect the changes in signal structure. The dynamic parameters of the SG filter can reduce any lost amplitude to make the denoised signals closer to the real signals. Therefore, additional research is needed to improve and enhance noise reduction algorithms in the future.

\section{Acknowledgments}

This work was supported in part by the JSPS KAKENHI Grant Numbers 19H04507.

\section{Conflicts of Interest}

The authors declare no conflict of interest.

\section{References}

[1] D. W. Jackson, T. M. Simon, and H. M. Aberman, "Symptomatic Articular Cartilage Degeneration: The Impact in the New Millennium,” Clinical Orthopaedics and Related Research, vol. 391, pp. 14-25, October 2001.

[2] A. Balajee and R. Venkatesan, "A Survey on Classification Methodologies Utilized for Classifying the Knee Joint Disorder Levels Using Vibroarthrographic Signals," Materials Today: Proceedings, in press.

[3] D. B. Percival and A. T. Walden, Wavelet Methods for Time Series Analysis, Cambridge: Cambridge University Press, 2010.

[4] R. Gong, K. Hase, H. Goto, K. Yoshioka, and S. Ota, "Knee Osteoarthritis Detection Based on the Combination of Empirical Mode Decomposition and Wavelet Analysis," Journal of Biomechanical Science and Engineering, vol. 15, no. 3, 20-00017, 2020.

[5] R. James, H. Appukuttan, and L. A. Joseph, "Mixed Noise Removal by Processing of Patches," Proceedings of Engineering and Technology Innovation, vol. 17, pp. 32-41, January 2021.

[6] R. Gong, H. Ohtsu, K. Hase, and S. Ota, "Vibroarthrographic Signals for the Low-Cost and Computationally Efficient Classification of Aging and Healthy Knees,” Biomedical Signal Processing and Control, vol. 70, 103003, September 2021.

[7] A. Sundar, V. Pahwa, and C. Das, “A New Method for Denoising Knee Joint Vibroarthrographic Signals," Annual IEEE India Conference, pp. 1-5, December 2015.

[8] Y. T. Chiang, C. H. Lu, C. C. Tuan, T. F. Lee, Y. C. Huang, and M. C. Chen, "Non-Invasive Detection of Sound Signals for Diagnosis of Ligament Injuries around Knee Based on Mel-Frequency Cepstrum,” Intelligent Systems and Applications, vol. 274, pp. 1940-1949, 2015.

[9] J. Rahul, M. Sora, and L. D. Sharma, “An Overview on Biomedical Signal Analysis,” International Journal of Recent Technology and Engineering, vol. 7, no. 5, pp. 206-209, January 2019.

[10] M. E. Torres, M. A. Colominas, G. Schlotthauer, and P. Flandrin, “A Complete Ensemble Empirical Mode Decomposition with Adaptive Noise,” IEEE International Conference on Acoustics, Speech, and Signal Processing, pp. 4144-4147, May 2011.

[11] P. A. Vikhar, "Evolutionary Algorithms: A Critical Review and Its Future Prospects," International Conference on Global Trends in Signal Processing, Information Computing, and Communication, pp. 261-265, December 2016. 
[12] R. Gong and K. Hase, "A Plant Root System Algorithm Based on Swarm Intelligence for One-Dimensional Biomedical Signal Feature Engineering,” https://arxiv.org/ftp/arxiv/papers/2108/2108.00214.pdf, July 31, 2021.

[13] L. Li, Y. Ma, B. Wang, H. Dong, and Z. Zhang, "Research on Traffic Signal Timing Method Based on Ant Colony Algorithm and Fuzzy Control Theory,” Proceedings of Engineering and Technology Innovation, vol. 11, pp. 21-29, January 2019.

[14] S. Ota, A. Ando, Y. Tozawa, T. Nakamura, S. Okamoto, T. Sakai, et al., "Preliminary Study of Optimal Measurement Location on Vibroarthrography for Classification of Patients with Knee Osteoarthritis," Journal of Physical Therapy Science, vol. 28, no. 10, pp. 2904-2908, 2016.

[15] R. Gong, K. Hase, H. Goto, and K. Yoshioka, "Post-Processing Algorithm for Removing Soft-Tissue Movement Artifacts from Vibroarthrographic Knee-Joint Signal,” 42nd Annual International Conference of the IEEE Engineering in Medicine and Biology Society, pp. 936-939, July 2020.

[16] N. Befrui, J. Elsner, A. Flesser, J. Huvanandana, O. Jarrousse, T. N. Le, et al., "Vibroarthrography for Early Detection of Knee Osteoarthritis Using Normalized Frequency Features,” Medical and Biological Engineering and Computing, vol. 56, no. 8, pp. 1499-1514, August 2018.

[17] R. Mancini and B. Carter, Op Amps for Everyone, United States: Elsevier, 2009.

[18] B. Boashash, Time-Frequency Signal Analysis and Processing: A Comprehensive Reference, London: Elsevier, 2016.

[19] Z. Wu and N. E. Huang, "Ensemble Empirical Mode Decomposition: A Noise Assisted Data Analysis Method," Advances in Adaptive Data Analysis, vol. 1, no. 1, pp. 1-41, January 2009.

[20] G. F. Inbar, O. Paiss, J. Allin, and H. Kranz, "Monitoring Surface EMG Spectral Changes by the Zero Crossing Rate," Medical and Biological Engineering and Computing, vol. 24, no. 1, pp. 10-18, 1986.

[21] M. Tanweer and K. A. Halonen, "Development of Wearable Hardware Platform to Measure the ECG and EMG with IMU to Detect Motion Artifacts," IEEE 22nd International Symp. on Design and Diagnostics of Electronic Circuits and Systems, pp. 1-4, April 2019.

[22] R. Schafer, "What is a Savitzky-Golay Filter?” IEEE Signal Processing Magazine, vol. 28, no. 4, pp. 111-117, July 2011.

[23] S. Dubnov, "Generalization of Spectral Flatness Measure for Non-Gaussian Linear Processes," IEEE Signal Processing Letters, vol. 11, no. 8, pp. 698-701, August 2004.

[24] K. Socha and M. Dorigo, “Ant Colony Optimization for Continuous Domains,” European Journal of Operational Research, vol. 185, no. 3, pp. 1155-1173, March 2008.

[25] J. Ning, Q. Zhang, C. Zhang, and B. Zhang, “A Best-Path-Updating Information-Guided Ant Colony Optimization Algorithm,” Information Sciences, vol. 433, pp. 142-162, April 2018.

[26] S. Poornachandra and N. Kumaravel, "Subband-Adaptive Shrinkage for Denoising of ECG Signals," EURASIP Journal on Advances in Signal Processing, vol. 2006, 081236, December 2006.

[27] N. Tanaka and M. Hoshiyama, “Articular Sound and Clinical Stages in Knee Arthropathy,” Journal of Musculoskeletal Research, vol. 14, no. 1, 1150006, March 2011.

[28] B. H. Tracey and E. L. Miller, "Nonlocal Means Denoising of ECG Signals,” IEEE Transactions on Biomedical Engineering, vol. 59, no. 9, pp. 2383-2386, September 2012.

[29] C. Kaur, A. Bisht, P. Singh, and G. Joshi, "EEG Signal Denoising Using Hybrid Approach of Variational Mode Decomposition and Wavelets for Depression,” Biomedical Signal Processing and Control, vol. 65, 102337, March 2021.

[30] G. Tzanetakis, G. Essl, and P. Cook, “Audio Analysis Using the Discrete Wavelet Transform,” WSEAS International Conference on Acoustics and Music: Theory and Applications, pp. 318-323, September 2001.

Copyright $($ by the authors. Licensee TAETI, Taiwan. This article is an open access article distributed under the terms and conditions of the Creative Commons Attribution (CC BY-NC) license (https://creativecommons.org/licenses/by-nc/4.0/). 